Reviews and perspectives

\title{
The suppression of repetition enhancement: A review of fMRI studies
}

\author{
Katrien Segaert $^{\mathrm{a}, \mathrm{b}, 1}$, Kirsten Weber ${ }^{\mathrm{b}, 1}$, Floris P. de Lange ${ }^{\mathrm{b}}$, Karl Magnus Petersson ${ }^{\mathrm{a}, \mathrm{b}}$, Peter Hagoort ${ }^{\mathrm{a}, \mathrm{b}, *}$ \\ a Max Planck Institute for Psycholinguistics, Nijmegen, the Netherlands \\ ${ }^{\mathrm{b}}$ Radboud University Nijmegen, Donders Institute for Brain, Cognition and Behaviour, Centre for Cognitive Neuroimaging, Nijmegen, the Netherlands
}

\section{A R T I C L E I N F O}

\section{Article history:}

Received 14 February 2011

Received in revised form

5 November 2012

Accepted 6 November 2012

Available online 14 November 2012

Keywords:

fMRI adaptation

Repetition suppression

Repetition enhancement

Priming

\begin{abstract}
A B S T R A C T
Repetition suppression in fMRI studies is generally thought to underlie behavioural facilitation effects (i.e., priming) and it is often used to identify the neuronal representations associated with a stimulus. However, this pays little heed to the large number of repetition enhancement effects observed under similar conditions. In this review, we identify several cognitive variables biasing repetition effects in the BOLD response towards enhancement instead of suppression. These variables are stimulus recognition, learning, attention, expectation and explicit memory. We also evaluate which models can account for these repetition effects and come to the conclusion that there is no one single model that is able to embrace all repetition enhancement effects. Accumulation, novel network formation as well as predictive coding models can all explain subsets of repetition enhancement effects.
\end{abstract}

(c) 2012 Elsevier Ltd. All rights reserved.

\section{Contents}

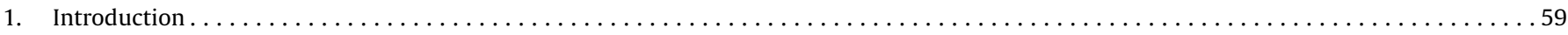

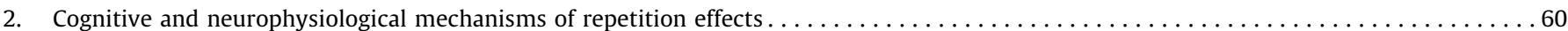

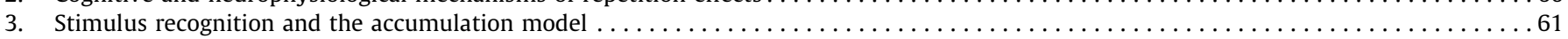

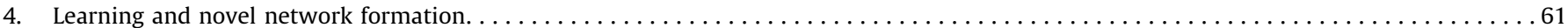

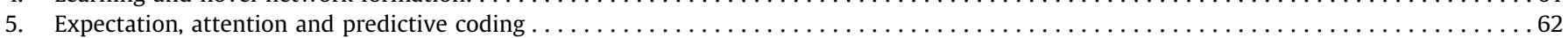

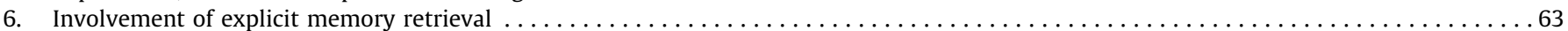

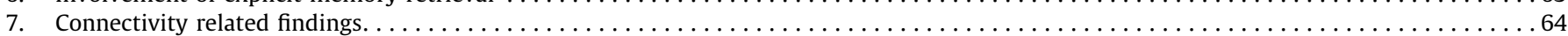

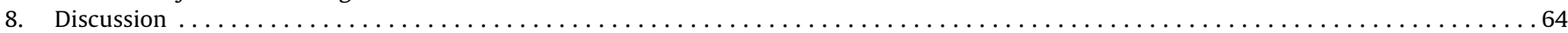

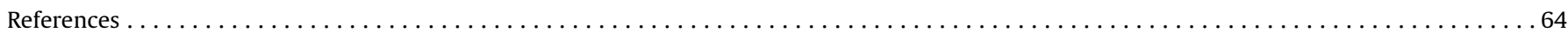

\section{Introduction}

In recent years repetition suppression in fMRI studies has been used to detect the nature of the neural code for perception and other forms of cognition. However, under similar circumstances, repetition enhancement instead of suppression is sometimes obtained. This complicates matters substantially, especially since these repetition enhancement effects are less well understood than repetition suppression effects. In this review we aim to contribute to a better understanding of repetition enhancement effects in the BOLD response, discussing the conditions under

\footnotetext{
* Correspondence to: Kapittelweg 29, 6525 EN Nijmegen, the Netherlands

Tel.: +3124 3610648; fax: +31243610652.

E-mail address: Peter.Hagoort@donders.ru.nl (P. Hagoort).

${ }^{1}$ The first two authors contributed equally to this work.
}

which these effects can be found and their possible underlying mechanisms and processes.

Repetition suppression is the reduction of neural responses to the repetition of stimulus features (Grill-Spector, Henson, \& Martin, 2006; Henson, 2003; Krekelberg, Boynton, \& van Wezel, 2006; Miller, Li, \& Desimone, 1991). In fMRI studies repetition suppression is sometimes called fMRI adaptation (Ganel et al., 2006; Grill-Spector \& Malach, 2001). Repetition suppression is assumed to be the neural correlate of behavioural priming in experiments that aim at uncovering implicit, automatic responses to stimuli or stimulus features (Henson, 2003; Wiggs \& Martin, 1998); whether these are immediate repetition effects or extend up to minutes, hours or days. Repetition suppression in the BOLD response is generally thought of as an effect of stimulus repetition per se, occurring independently of other psychological or neurophysiological variables. The appeal of repetition suppression paradigms lies in its potential superiority over standard fMRI 

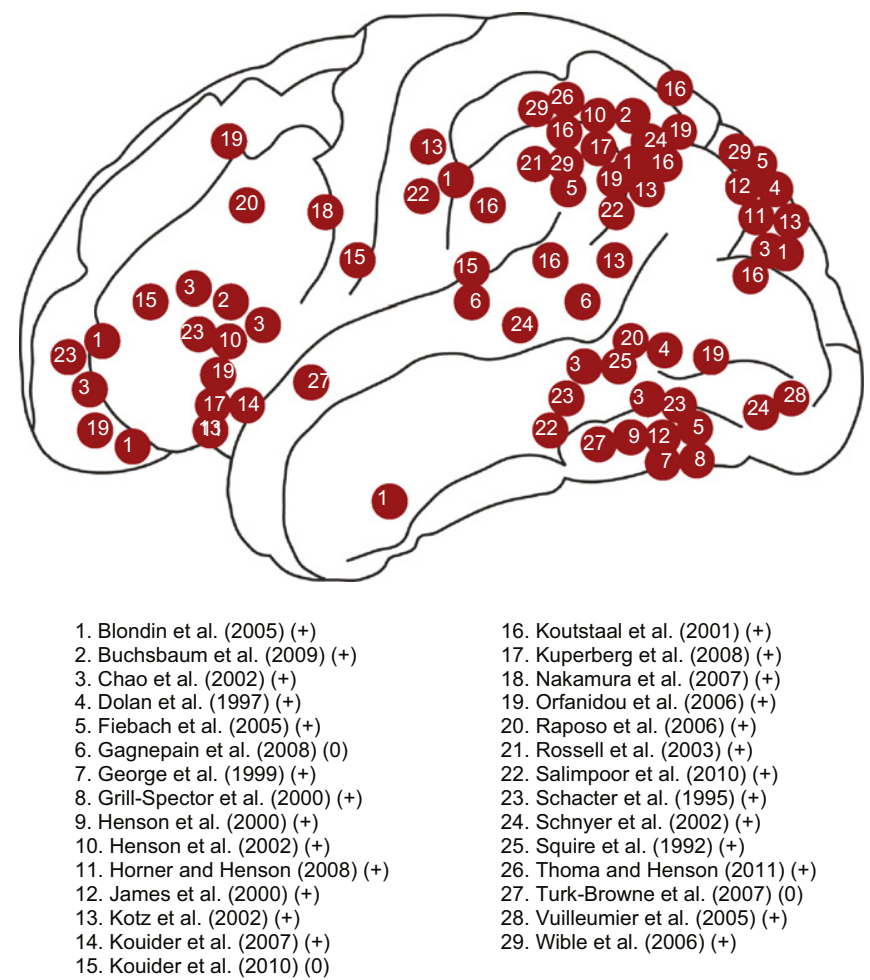

16. Koutstaal et al. (2001) (+)

17. Kuperberg et al. $(2008)(+)$

18. Nakamura et al. (2007) $(+)$

19. Orfanidou et al. (2006) $(+)$

20. Raposo et at. (2006) (+)

20. Raposo et al. (2006) (+)

21. Rossell etal. (2003) (+)

22. Salimpoor et al. (2010) (+)

23. Schacter et al. (1995) (+)

24. Schnyer et al. $(2002)(+)$

25. Squire et al. (1992) (+)

26. Thoma and Henson (2011) $(+)$

27. Turk-Browne et al. (2007) $(0)$

28. Vuilleumier et al. $(2005)(+)$

29. Wible et al. (2006) (+)

Fig. 1. Localization of repetition enhancement effects. The figure illustrates a coarse localization of enhancement effects in the studies reviewed in this paper in a glass brain. The numbers refer to the references underneath. Additionally, in the references $(0)$ indicates that no behavioural priming effect was observed and $(+)$ indicates that a behavioural facilitation effect was found.

paradigms with regard to tapping into functional specificity of neuronal populations even within one voxel (Grill-Spector \& Malach, 2001; Krekelberg et al., 2006). It is therefore a valuable tool in cognitive neuroimaging research, from visual (Grill-Spector et al., 1999; Loffler, Yourganov, Wilkinson, \& Wilson, 2005) to language processing (Gagnepain et al., 2008; Glezer, Jiang, \& Riesenhuber, 2009). However, a more detailed look at studies of repetition effects reveals that frequently increases in the BOLD response or repetition enhancement is observed. Like repetition suppression, these enhancement effects have been found all over the brain (see Fig. 1). Repetition enhancement has received strikingly less attention than repetition suppression. We argue that it is important to start considering these effects more closely and evaluate the full range of neural response profiles that may accompany stimulus repetition. We will review cases when the stimulus is identically repeated (i.e. identity priming) as well as cases when only one property of the stimulus is repeated (e.g. semantic priming: only some semantic features of a linguistic stimulus are repeated between prime and target).

Repetition enhancement, as well as suppression, reflects interesting changes in information processing. The main objective of this review is to delineate the conditions under which repetition enhancement rather than suppression can be observed and discuss the possible underlying cognitive and neural mechanisms.

\section{Cognitive and neurophysiological mechanisms of repetition effects}

In this review we will discuss several cognitive mechanisms that we believe to be important in determining the direction of repetition effects in fMRI studies (i.e. repetition enhancement vs. suppression). These mechanisms are stimulus recognition, learning, expectation, attention and explicit memory. We will address these in detail in separate sections of this review.

Several models have been proposed to explain repetition effects in the BOLD response. These models specify mechanisms at the level of a neural population.

Several of these neurophysiological mechanisms are able to explain repetition suppression effects: the fatigue model, the sharpening model, the accumulation model and the predictive coding model (see Grill-Spector et al. (2006) for an extensive review). Fatigue models explain repetition suppression in terms of neural populations which in general respond less to repeated stimuli, for example, by firing rate adaptation (Grill-Spector \& Malach, 2001; Miller et al., 1991). Sharpening models propose that fewer neurons (more specifically, only those neurons coding for essential stimulus information) respond to repeated stimuli. The stimulus representation becomes sharpened so the overall BOLD response is suppressed (Desimone, 1996; Miller et al., 1991; Wiggs \& Martin, 1998). According to accumulation (or facilitation) models (James \& Gauthier, 2006), priming makes the peak of neural activity shift closer to the event that triggered the activity, as the behavioural response is faster. Consequently, in priming conditions there is less cumulative activity and thus a reduced BOLD response. The fourth and last group of models, predictive coding models, propose that sensory evidence is interpreted in the context of subjective biases and statistical accounts of past experience (Friston, 2005). One of the key brain mechanisms is the prediction of upcoming events in the environment, while prediction error (the difference between what was predicted and what actually occurred) is used to update perceptual inference. Prediction of stimulus repetition reduces the prediction error which leads to repetition effects in the BOLD response. There is value in all of these models since it is unlikely that there is one single underlying mechanism encompassing all repetition suppression effects (Grill-Spector et al., 2006).

Similarly to repetition suppression, several different neurophysiological accounts can explain repetition enhancement: the accumulation model (James \& Gauthier, 2006), the predictive coding model (Friston, 2005) and novel network formation (Henson, Shallice, \& Dolan, 2000). Moreover, the involvement of explicit memory retrieval is sometimes accompanied by repetition enhancement effects (Henson, 2003). We will argue that it is unlikely that there is one single underlying mechanism encompassing all repetition enhancement effects. Much like it is the case for repetition suppression (Grill-Spector et al., 2006), repetition enhancement is not a unitary phenomenon. In the next sections of this review, we will discuss the mechanisms and processes that are able to explain repetition enhancement. Accumulation and predictive coding models are able to explain suppression effects as well as enhancement effects. Novel network formation and the involvement of explicit memory are processes that are able to explain repetition enhancement but not suppression effects.

In the next sections of this review we will also revisit the complex relationship between cognitive psychological mechanisms and the mechanisms explaining BOLD effects. We will argue that the accumulation model is relevant when stimulus recognition plays a role, novel network formation is relevant when learning plays a role, and the predictive coding model is relevant when attention or expectation plays a role. Furthermore, explicit memory retrieval is sometimes involved in implicit memory tasks.

Repetition effects should ultimately not only be explained at the level of a group of neurons, but also at the level of individual neurons. However, at the level of individual neurons the underlying neurophysiologic mechanisms and their interactions are not yet well understood. This complicates our understanding of when and why repetition enhancement versus suppression occurs. A diverse range of plasticity mechanisms are possible candidates 
for causing neural repetition suppression and enhancement: for example firing-rate adaptation (Desimone, 1996; Li, Miller, \& Desimone, 1993), long-term potentiation (LTP) and long-term depression (LTD) (Cooke \& Bliss, 2006; Kim \& Linden, 2007). However, how they interact in bringing about suppression or enhancement effects in the BOLD response, requires additional research.

It should also be noted that the relationship between the neural mechanisms at the level of individual neurons and the mechanisms at the level of the BOLD effect is complicated by the poor temporal resolution of fMRI. With fMRI we can only capture the net activity of a group of neurons; neuronal suppression and enhancement might be integrated without taking the respective latencies of these effects into consideration. Importantly, from studies using measures with a better temporal resolution (e.g. magnetoencephalography (MEG) and event-related potentials (ERPs)), we know that repetition effects can move from initial repetition enhancement to repetition suppression in a later time window, even within the same region (Marinkovic et al., 2003; Petit, Midgley, Holcomb, \& Grainger, 2006) as well as from repetition suppression to repetition enhancement (Henson, Mouchlianitis, Matthews, \& Kouider, 2008). A caveat of fMRI studies is that the BOLD response may reflect the net result of these processes. The net result could be the absence of any repetition effect or a bias towards either suppression or enhancement that does not reflect the diversity of the neural patterns.

How do repetition effects measured in fMRI relate to the behavioural priming effects? There is a general assumption that repetition suppression in the BOLD response is accompanied by facilitation in behavioural performance (Wig, Grafton, Demos, \& Kelley, 2005). A logical extension of this idea, would be that repetition enhancement must be accompanied by inhibition in behavioural performance. But this is by no means the rule. There is a wide range of possible relations between repetition effects and behavioural priming effects. In some cases when repetition enhancement is observed, no behavioural effect (Gagnepain et al., 2008; Kouider, de Gardelle, Dehaene, Dupoux, \& Pallier, 2010; Turk-Browne, Yi, Leber, \& Chun, 2007) or inhibition is found (Egner \& Hirsch, 2005). However, more generally repetition enhancement effects are accompanied by behavioural facilitation effects (see also Fig. 1). Moreover, in some studies the size of the repetition enhancement effects is correlated to the size of the behavioural improvement (Salimpoor, Chang, \& Menon, 2010; Thoma \& Henson, 2011) giving evidence of a direct link between repetition enhancement effects and behavioural facilitation effects.

We will now turn to the cognitive and neurophysiological mechanisms that we believe to be important in determining the direction of repetition effects in fMRI studies.

\section{Stimulus recognition and the accumulation model}

The first condition under which repetition enhancement in the BOLD response is frequently observed is when perceptual stimulus visibility is manipulated by degradation or masking. Such manipulations of stimulus visibility affect recognition. Repeated exposure to stimuli with low visibility generally leads to increased perceptual performance and is accompanied by repetition enhancement effects in the brain. Higher performance in recognizing degraded objects (Dolan et al., 1997) or in identifying degraded famous faces (George et al., 1999) when they were preceded by corresponding non-degraded images was accompanied by enhanced activation in fusiform gyrus. In a scene selective region (parahippocampal place area) there was suppression for the repetition of the same high-visibility scene and enhancement for the repetition of the same low-visibility scene (Turk-Browne et al., 2007). Repetition enhancement has furthermore been observed in masked conditions. Briefly presented and masked objects could initially not be recognized but a 5-7-day period of training increased performance and was associated with enhanced activation in two object-selective regions (lateral occipital complex and fusiform gyrus) (Grill-Spector, Kushnir, Hendler, \& Malach, 2000).

Repetition enhancement effects in cases in which the target was masked or degraded can be understood in the light of the accumulation model (James \& Gauthier, 2006). According to the accumulation model, neuronal activity peaks earlier when primed compared to unprimed targets are processed. Using a gradual unmasking technique James, Humphrey, Gati, Menon, and Goodale (2000) showed that repeated exposure resulted in increasing activation before and decreasing activation after object recognition. In their experiment, primes were presented in the first phase of the experiment and (unprimed and primed) targets in the second phase. Primed objects were recognized sooner than unprimed objects and there were steeper rises in activation and earlier activation peaks for primed objects. Before recognition, primed relative to unprimed objects led to enhanced activation; yet after recognition, primed objects led to reduced activation. Thus, if the probability of successfully recognizing a stimulus greatly increases when primed (e.g. for stimuli with low visibility or masked stimuli), repetition enhancement instead of suppression may occur. In fact, increased object recognition is generally accompanied by increased cortical activation (Bar et al., 2001; Malach et al., 1995; Ress \& Heeger, 2003). Furthermore, for repeated items of which participants had no recollection (because participants had been instructed to ignore them when they were first presented), there was a behavioural advantage on real-object decisions which was associated with enhancement effects in bilateral lingual gyri (Vuilleumier, Schwartz, Duhoux, Dolan, \& Driver, 2005).

At times repetition enhancement in the BOLD response is observed when the prime (not the target) is masked. This was the case for some of the language studies investigating masked word priming. Subliminal exposure to a word facilitates subsequent conscious processing of this word. This increased behavioural performance was accompanied by repetition enhancement effects in parietal and left temporal regions (Nakamura, Dehaene, Jobert, Le Bihan, \& Kouider, 2007; Schnyer, Ryan, Trouard, \& Forster, 2002). However, in other studies on masked word priming repetition suppression effects were found in very similar regions (Dehaene et al., 2001; Nakamura et al., 2007). It has been suggested (Schnyer et al., 2002) that, for word priming with masked primes, repetition enhancement effects are observed if activation from the masked prime continues to spread until the target is identified. Activation from the prime is then added to activation elicited by the target itself.

Future studies on word priming with masked primes will be needed to elucidate why in some cases repetition enhancement is observed and in others repetition suppression. We suggest that the task (Nakamura et al., 2007) or the stimulus interval could play a role in determining the outcome (see Section 5).

\section{Learning and novel network formation}

The second parameter to consider is learning by repetition of novel stimuli. Novel stimuli that have not been encountered before by the participant do not have a stable memory representation. Therefore, repeating novel or unfamiliar items generally leads to repetition enhancement effects in the BOLD response. This has been found for novel symbols (Henson et al., 2000), faces 
(Henson et al., 2000), objects (Gruber \& Muller, 2005; James et al., 2002; Schacter et al., 1995) and pseudowords (Fiebach, Gruber, \& Supp, 2005; Gagnepain et al., 2008; Henson, 2001; Kouider et al., 2010). The task however may play an additional role in determining the repetition effects for novel stimuli (Henson, Shallice, Gorno-Tempini, \& Dolan, 2002) (see Section 5). Behaviourally, the repetition of both familiar as well as unfamiliar items leads to a priming effect (Henson et al., 2000; Salasoo, Shiffrin, \& Feustel, 1985; Schacter et al., 1995, although see Gagnepain et al., 2008) but the effect is typically stronger for familiar items.

The repetition enhancement effect in these cases is assumed to reflect "novel network formation" (Henson et al., 2000), the creation of a new representation. Novel network formation is the process of building up a neural network coding for the novel stimulus. After stimulus presentation certain neurons together will code for this novel item. At the first encounter of an unfamiliar item, the BOLDresponse and firing rates are increased compared to familiar items. At this point, the representation is unstable with a large tuning curve and a large number of neurons responding to the novel item. Moreover, repetition of this novel item will lead to the repetition enhancement response described above. Within these experiments, repeated novel items are still considered novel, while items termed 'familiar' have a well-established long-term memory representation. Only through repeated encounters and most probably consolidation (see next paragraph) will a novel item be learned and eventually become familiar.

It would then follow that once a representation has been built, repeating these stimuli should lead to repetition suppression instead of enhancement, as no additional memory trace formation would be necessary. Once familiar, an items' tuning curve is more narrow and a more sharpened neural representation is established (Rainer \& Miller, 2000; Ranganath \& D'Esposito, 2001; Ranganath \& Rainer, 2003). This sharpening of the neural representation results in an overall net effect of repetition suppression in the BOLD response (Desimone, 1996; Miller et al., 1991; Wiggs \& Martin, 1998). Sharpening or tuning of representations can be conceived as complementary mechanisms to the formation of representations. However, to our knowledge, a switch from repetition enhancement to suppression in the context of these types of fMRI studies has so far not been found. Henson et al. (2000) and Conrad, Giabbiconi, Muller, and Gruber (2007) observed continuously increasing versus decreasing neural activity for up to ten repetitions of novel versus familiar objects, but in these studies the enhancement response did not switch into suppression after a certain number of repetitions. The number of repetitions might have been too few to detect such a switch. Furthermore, it is possible that the "novel network formation" mechanism needs consolidation before an object can become "familiar", which might only be realised during sleep (Walker \& Stickgold, 2006). Supporting this idea, Davis et al. found reduced activations to novel words learned on the previous day, while no such response was found for words learned on the same day (Davis, Di Betta, MacDonald, \& Gaskell, 2008).

\section{Expectation, attention and predictive coding}

Repetition effects are not purely automatic processes, triggered by particular types or sequences of stimuli. Rather than being a passive, stimulus-driven device 'waiting' to be activated by sensations, the brain is continuously and actively filtering, attending to and interpreting the information provided by our senses (Friston, 2005). In line with this, it is becoming increasingly clear that "top down" cognitive factors like expectation and attention play an important role in determining the amount and direction of repetition effects in the BOLD response.
Predictive coding models propose that sensory evidence is interpreted in the context of subjective biases that are established on the basis of statistical regularities in the outside world (Friston, 2005). In this view, our percepts and decisions arise from the interplay between externally generated input and internally generated hypotheses, expectations and interpretations (Friston, 2005; Mesulam, 2008). According to these models, one of the key brain mechanisms is the prediction of upcoming events in the environment (Bar, 2007; Friston, 2005; Rao \& Sejnowski, 2002). The prediction error (the difference between what was predicted and what actually occurred) is used to update perceptual inferences. Therefore, fewer neuronal resources become engaged by accurately inferred events. Within such a model prior knowledge about the statistics of the world predict stimulus repetition by default, thereby reducing the prediction error because of topdown expectations, which leads to the repetition suppression effect in the brain and the behavioural priming response (Friston, 2005).

From predictive coding models it follows that the extent to which repetition is expected can modulate the size of repetition suppression effects. Indeed, when the probability and hence the participant's expectation of repetitions was reduced, repetition suppression for repeated faces in an fMRI study was diminished in the fusiform face area (Summerfield, Trittschuh, Monti, Mesulam, \& Egner, 2008; see also, Summerfield, Wyart, Johnen, \& de Gardelle, 2011), and repetition suppression for repeated tones in an MEG study was diminished in the auditory cortex (Todorovic, Van Ede, Maris, \& de Lange, 2011). Repetition effects in the BOLD response are furthermore modulated by the meaningfulness of stimuli: meaningfulness reduces the prediction error. Repetition effects in the BOLD response are smaller when objects are less meaningful (e.g. "impossible" in 3-D) (Schacter et al., 1995) or when there is no functional experience with the stimuli (Weisberg, van Turennout, \& Martin, 2007).

Can the size of repetition effects in the BOLD response also be modulated by attention? It is well-known that selective attention can "boost" neural activity in the relevant brain regions (Corbetta \& Shulman, 2002). Activity increases related to attention have been demonstrated in sensory processes such as visual perception (Corbetta, Miezin, Dobmeyer, Shulman, \& Petersen, 1990; Silver, Ress, \& Heeger, 2007) but also action generation (Rowe, Friston, Frackowiak, \& Passingham, 2002) and intention (Lau, Rogers, Haggard, \& Passingham, 2004). Given that repetition effects are often subtle, they are likely to be more visible when selective attention boosts the overall stimulus-induced activity. Indeed, several studies have shown that repetition suppression effects in the BOLD response are much stronger, and perhaps even fully dependent on the presence of selective attention (Eger, Henson, Driver, \& Dolan, 2004; Murray \& Wojciulik, 2004; Yi \& Chun, 2005). In a similar vein, stimuli that were emotionally salient evoked substantially larger repetition suppression effects (Ishai, Pessoa, Bikle, \& Ungerleider, 2004). Furthermore, Larsson and Smith (2011) demonstrated that the effect of expectation is attention dependent. The effect of expectation on repetition suppression disappeared when attention was diverted away from the stimuli. The results on the influence of attention on repetition suppression effects in the BOLD response can be understood in the framework of predictive coding models: when attention is directed towards the interpretation of particular stimuli, more accurate predictions can be inferred from them and the prediction error can be minimized.

Accordingly, cognitive factors like expectation and attention can modulate the strength of repetition suppression effects in the brain. But can these factors also reverse repetition suppression and produce enhancement? There are good theoretical reasons to believe manipulations of expectation could lead to enhancement 
effects but this has yet to be tested. Our perceptual world is generally highly stable so our perceptual system may have a relatively strong default to expect repetition. A straightforward prediction from predictive coding models is that if a particular change is more likely than a repetition, repetition should lead to enhancement effects. This could be empirically tested (but to the best of our knowledge has so far not been tested) by providing participants with visual input that is changing in a predictable fashion, rather than repeating. It will be interesting to see whether repetition suppression can be reversed by extensively training participants to expect such a changing environment ( $\mathrm{Li} \&$ DiCarlo, 2008).

Some studies suggest that also manipulations of attention could bring about repetition enhancement versus suppression effects. Differences in task demands (and thereby, differences in the attention state of the participant) appear to modulate the direction of repetition effects. For masked word priming, the parietotemporal cortex showed repetition suppression for a semantic categorization task and repetition enhancement for a reading aloud task (Nakamura et al., 2007). The same stimuli and masking procedure were used in both tasks. Reading but not semantic categorization relied on reciprocal excitatory functional connections between parietal and temporal regions. Nakamura et al. (2007) put forward two potential explanations for this top down influence of the task demands of reading aloud: firstly, additional cognitive processes like self-monitoring during reading aloud may be responsible for the enhancement effect, or secondly, the attention task demands of reading aloud may be higher than of semantic categorization (the authors argue that reading aloud is not fully automatic and may require central attention). Likewise, phonological priming (i.e. words are primed by phonologically related letter strings) has been associated with repetition enhancement in regions implicated in phonological and articulatory processing (Kouider, Dehaene, Jobert, \& Le Bihan, 2007). In this study, the phonological priming condition was the only priming condition in which the letter string prime was recognizable as a misspelled word. This was not the case for the letter string prime in the other priming conditions (unrelated priming or orthographic priming). In this way, the phonological priming condition under which enhancement was observed deviated from the other conditions, leading the authors to suggest that attention might have played a role. The functional explanation for the effects in the two above mentioned studies (Kouider et al., 2007; Nakamura et al., 2007) is still rather unclear, but we want to point out that in both cases enhancement effects in the BOLD response were observed for conditions in which participants' attention was directed to the stimulus more than for other conditions within the study.

Taken together, the results of these studies suggest that the size and possibly the direction of repetition effects are influenced by the type of attention required by the specific task at hand. It has also been shown that predictability can interact with attention in bringing about suppression versus enhancement effects. For example, while the hippocampus shows repetition suppression when stimulus repetitions are incidental to the task at hand (Kumaran \& Maguire, 2006, 2009), the same region shows repetition enhancement when the stimuli are task-relevant to the participant (Brown \& Aggleton, 2001). We would furthermore like to note that the contribution of "top down" cognitive factors like expectation and attention can also depend on stimulus timing. Stimulus onset asynchronies, the time intervals between two stimulus presentations, seem to influence repetition effects in the BOLD response, in size (Henson et al., 2000; van Turennout, Ellmore, \& Martin, 2000) and direction (compare the repetition enhancement effects in Kuperberg, Lakshmanan, Greve, and West (2008) to the repetition suppression effects in Rossell, Price, and
Nobre (2003) and Wible et al. (2006)). Depending on the stimulus interval, the engagement of certain cognitive processes like attention, expectation and even explicit memory (see next section) may be different.

\section{Involvement of explicit memory retrieval}

Unlike the cases we described in previous sections, the repetition enhancement effects described in this section are effects in addition to (not instead of) suppression effects. This is in line with the idea that repetition enhancement effects in the BOLD response might reflect some additional process on the target (Henson, 2003), for instance explicit memory retrieval. Priming effects are generally assessed with implicit memory tasks (Roediger \& McDermott, 1993), which do not require the conscious recollection of particular previous experiences. But even when recollection is not required for the task at hand, participants may recollect previous encounters with the stimulus, whether intentionally or incidentally. For instance, participants might become aware of similarities between primes and targets and even make same-different judgments on primes and targets. This might result in repetition enhancement effects in regions responsible for making these same-different judgements in addition to suppression effects in regions responsible for processing the stimulus per se.

Support for this idea is found in one of the first neuroimaging studies of priming (Squire et al., 1992). In this positron-emission tomography (PET) study the primes were presented prior to the scanning and a blood flow increase in the right hippocampal region was reported in addition to a decrease in right posterior regions, thus evidencing a role for explicit retrieval, probably incidental or non-intentional (Schacter \& Buckner, 1998). Ever since, many studies have accumulated evidence for a role of explicit memory as a process in priming tasks. In visual object priming studies for instance, the behavioural priming effect is generally associated with suppression in object-responsive regions and in a number of cases at the same time with repetition enhancement in frontal, posterior and hippocampal regions (Blondin \& Lepage, 2005; Chao, Weisberg, \& Martin, 2002; Horner \& Henson, 2008; Koutstaal et al., 2001), regions known to be involved in memory retrieval (McDermott et al., 1999; Rugg, Otten, \& Henson, 2002; Schott et al., 2005, 2006; Wagner, Shannon, Kahn, \& Buckner, 2005). Not only in visual object priming studies but also in language priming studies, repetition enhancement effects in these brain regions seem to reflect the involvement of explicit memory processes. While word priming and semantic priming studies generally show repetition suppression in regions processing lexical and conceptual aspects of words, these studies occasionally also show enhancement effects in regions showing great overlap with explicit memory regions (Kotz, Cappa, von Cramon, \& Friederici, 2002; Kuperberg, et al., 2008; Orfanidou, Marslen-Wilson, \& Davis, 2006; Raposo, Moss, Stamatakis, \& Tyler, 2006; Rossell et al., 2003; Wible et al., 2006).

Additional support comes from studies by Schott et al. (2005, 2006), showing that regions involved with implicit priming are distinct from regions involved with explicit retrieval. Furthermore, in a priming study with a short-term memory recognition task requiring explicit recollection of previous experiences Buchsbaum and D'Esposito (2009) found repetition suppression in anterior temporal regions and enhancement in posterior temporal, dorsal frontal and parietal regions. This pattern of activation is very similar to the one found in priming studies with implicit tasks. These results, and similarly the results from other priming studies with explicit memory tasks (e.g. Druzgal \& D'Esposito, 2001), provide corroborating evidence for the idea that some of the enhancement effects in studies with an implicit 
Table 1

Summary of the different cognitive and neurophysiological mechanisms accompanied by repetition enhancement effects.

\begin{tabular}{|c|c|c|}
\hline Study characteristics & Cognitive mechanism & Neurophysiological mechanism \\
\hline Stimulus is degraded or masked (prime or target is masked) & Stimulus recognition & Accumulation model \\
\hline Stimulus is novel or unfamiliar & Learning & Novel network formation \\
\hline To be tested: stimulus repetitions are unexpected & Expectation & Predictive coding model \\
\hline To be tested: attention is directed to a particular stimulus/condition & Attention & Predictive coding model \\
\hline Implicit memory task with incidental or intentional explicit memory retrieval of the prime & Explicit memory & Additional activity in explicit memory regions \\
\hline
\end{tabular}

task reflect explicit memory retrieval. It remains an open question however, whether memory retrieval was intentional or incidental and which factors trigger participants to retrieve information about the prime.

\section{Connectivity related findings}

So far most attention has been devoted to stimulus based priming and repetition effects. However, repetition effects can also arise beyond stimulus based processing. This can be priming of stimulus-decision (Race, Shanker, \& Wagner, 2009; Wig, Buckner, \& Schacter, 2009) or stimulus-response mappings (Dobbins, Schnyer, Verfaellie, \& Schacter, 2004; Horner \& Henson, 2008; Race et al., 2009). These types of priming effects were revealed in repetition suppression (Dobbins et al., 2004; Horner \& Henson, 2008; Race et al., 2009) as well as enhancement effects in the BOLD response (Race et al., 2009; Salimpoor et al., 2010). Additionally, it is possible that during the mapping process, the regions showing these repetition effects strengthen their connection by functional connectivity, e.g. neural synchrony (Engel, Fries, \& Singer, 2001). There is indeed evidence that this is the case both for studies finding repetition suppression (Ghuman, Bar, Dobbins, \& Schnyer, 2008) as well as those finding repetition enhancement (Salimpoor et al., 2010). Thus, these results suggest that priming and repetition effects in the BOLD response can arise at the level of functional connectivity but further investigation is needed for a conclusive pattern on how repetition effects and functional connectivity are linked.

\section{Discussion}

Repeating stimuli results in behavioural changes and changes in the BOLD response. Changes in the BOLD response can be repetition suppression, but also repetition enhancement, or both (i.e., in different regions). FMRI adaptation paradigms have been used as a tool to identify neural populations encoding specific stimulus features. But we believe that this goal of specifying neural encoding properties can only be realised when taking into account the various cognitive factors modulating the size and direction of the repetition effects. Stimulus recognition, learning, attention, expectation and explicit memory influence repetition effects in the BOLD response. The underlying neurophysiological mechanisms might include predictive coding, accumulation and novel network formation. Furthermore, explicit memory retrieval seems to sometimes play a role in implicit memory tasks. In Table 1 we have summarized the cognitive and neurophysiological mechanisms that are accompanied by repetition enhancement effects in fMRI studies.

Each of these neurophysiological mechanisms accounts for enhancement effects that are not explained by another mechanism. It is thus unlikely one could propose a single new mechanism explaining all enhancement effects and accordingly each of these cognitive and neurophysiological mechanisms should be taken into consideration. One should also aim to look at repetition effects at different levels, from the cellular (single neuron) to the mesoscopic (neuronal populations) and large-scale systems level. This will yield better insights into how adaptive neuronal plasticity effects result in different net effects at a higher level and thus might cause different types of priming effects.

With appropriate and careful experimentation, fMRI adaptation paradigms can be used to measure neuronal adaptation and thus specific encoding properties. In many cases, repetition effects in the BOLD response reflect a combination of neuronal adaptation on the one hand and effects dependent on cognitive factors like e.g. attention or expectation on the other. These two elements can be dissociated experimentally. For instance, in the study by Larsson and Smith (2011), attention was turned away from the stimuli while holding the influence of expectation constant. More generally, empirical studies that orthogonally manipulate the cognitive psychological variables outlined above will be needed to test the predictions that arise from our present conclusions. The inherent limitations set by neuroimaging designs need to be overcome by integrating knowledge from different neuroimaging methods. While we believe that priming and repetition studies are useful to investigate cognitive processes, it cannot be taken for granted that repetition always leads to repetition suppression effects in the BOLD response. Instead, researchers need to carefully consider whether repetition suppression or repetition enhancement can be predicted based on the variables at hand.

\section{References}

Bar, M. (2007). The proactive brain: Using analogies and associations to generate predictions. Trends in Cognitive Sciences, 11(7), 280-289.

Bar, M., Tootell, R. B. H., Schacter, D. L., Greve, D. N., Fischl, B., Mendola, J. D., et al (2001). Cortical mechanisms specific to explicit visual object recognition. Neuron, 29(2), 529-535.

Blondin, F., \& Lepage, M. (2005). Decrease and increase in brain activity during visual perceptual priming: An fMRI study on similar but perceptually different complex visual scenes. Neuropsychologia, 43(13), 1887-1900.

Brown, M. W., \& Aggleton, J. P. (2001). Recognition memory: What are the roles of the perirhinal cortex and hippocampus? Nature Reviews Neuroscience, 2(1), 51-61.

Buchsbaum, B. R., \& D'Esposito, M. (2009). Repetition suppression and reactivation in auditory-verbal short-term recognition memory. Cerebral Cortex, 19(6), 1474-1485.

Chao, L. L., Weisberg, J., \& Martin, A. (2002). Experience-dependent modulation of category-related cortical activity. Cerebral Cortex, 12(5), 545-551.

Conrad, N., Giabbiconi, C. M., Muller, M. M., \& Gruber, T. (2007). Neuronal correlates of repetition priming of frequently presented objects: Insights from induced gamma band responses. Neuroscience Letters, 429(2-3), 126-130.

Cooke, S. F., \& Bliss, T. V. P. (2006). Plasticity in the human central nervous system. Brain, 129, 1659-1673.

Corbetta, M., Miezin, F. M., Dobmeyer, S., Shulman, G. L., \& Petersen, S. E. (1990) Attentional modulation of neural processing of shape, color, and velocity in humans. Science (New York, N.Y.), 248(4962), 1556-1559.

Corbetta, M., \& Shulman, G. L. (2002). Control of goal-directed and stimulus-driven attention in the brain. Nature Reviews Neuroscience, 3(3), 201-215.

Davis, M. H., Di Betta, A. M., MacDonald, M. J., \& Gaskell, M. G. (2008). Learning and consolidation of novel spoken words. Journal of Cognitive Neuroscience, 21(4) 803-820.

Dehaene, S., Naccache, L., Cohen, L., Le Bihan, D., Mangin, J. F., Poline, J. B., et al. (2001). Cerebral mechanisms of word masking and unconscious repetition priming. Nature Neuroscience, 4(7), 752-758.

Desimone, R. (1996). Neural mechanisms for visual memory and their role in attention. Proceedings of the National Academy of Sciences, 93, 13494-13499. 
Dobbins, I. G., Schnyer, D. M., Verfaellie, M., \& Schacter, D. L. (2004). Cortical activity reductions during repetition priming can result from rapid response learning. Nature, 428(6980), 316-319.

Dolan, R. J., Fink, G. R., Rolls, E., Booth, M., Holmes, A., Frackowiak, R. S. J., et al. (1997). How the brain learns to see objects and faces in an impoverished context. Nature, 389(6651), 596-599.

Druzgal, T. J., \& D’Esposito, M. (2001). Activity in fusiform face area modulated as a function of working memory load. Cognitive Brain Research, 10(3), 355-364.

Eger, E., Henson, R. N. A., Driver, J., \& Dolan, R. J. (2004). BOLD repetition decreases in object-responsive ventral visual areas depend on spatial attention. Journal of Neurophysiology, 92(2), 1241-1247.

Egner, T., \& Hirsch, J. (2005). Where memory meets attention: Neural substrates of negative priming. Journal of Cognitive Neuroscience, 17(11), 1774-1784.

Engel, A. K., Fries, P., \& Singer, W. (2001). Dynamic predictions: Oscillations and synchrony in top-down processing. [10.1038/35094565]. Nature Reviews Neuroscience, 2(10), 704-716.

Fiebach, C. J., Gruber, T., \& Supp, G. G. (2005). Neuronal mechanisms of repetition priming in occipitotemporal cortex: Spatiotemporal evidence from functional magnetic resonance imaging and electroencephalography. Journal of $\mathrm{Neu}$ roscience, 25(13), 3414-3422.

Friston, K. (2005). A theory of cortical responses. Philosophical Transactions of the Royal Society of London, 360(1456), 815-836.

Gagnepain, P., Chetelat, G., Landeau, B., Dayan, J., Eustache, F., \& Lebreton, K. (2008). Spoken word memory traces within the human auditory cortex revealed by repetition priming and functional magnetic resonance imaging. Journal of Neuroscience, 28(20), 5281-5289.

Ganel, T., Gonzalez, C. L. R., Valyear, K. F., Culham, J. C., Goodale, M. A., \& Kohler, S. (2006). The relationship between fMRI adaptation and repetition priming. Neuroimage, 32(3), 1432-1440.

George, N., Dolan, R. J., Fink, G. R., Baylis, G. C., Russell, C., \& Driver, J. (1999) Contrast polarity and face recognition in the human fusiform gyrus. Nature Neuroscience, 2(6), 574-580.

Ghuman, A. S., Bar, M., Dobbins, I. G., \& Schnyer, D. M. (2008). The effects of priming on frontal-temporal communication. Proceedings of the National Academy of Sciences, 105(24), 8405-8409.

Glezer, L. S., Jiang, X., \& Riesenhuber, M. (2009). Evidence for Highly Selective Neuronal Tuning to Whole Words in the "Visual Word Form Area". Neuron, 62(2), 199-204.

Grill-Spector, K., Henson, R., \& Martin, A. (2006). Repetition and the brain: Neural models of stimulus-specific effects. Trends in Cognitive Sciences, 10(1), 14-23.

Grill-Spector, K., Kushnir, T., Edelman, S., Avidan, G., Itzchak, Y., \& Malach, R. (1999). Differential processing of objects under various viewing conditions in the human lateral occipital complex. Neuron, 24(1), 187-203.

Grill-Spector, K., Kushnir, T., Hendler, T., \& Malach, R. (2000). The dynamics of object-selective activation correlate with recognition performance in humans. Nature Neuroscience, 3(8), 837-843.

Grill-Spector, K., \& Malach, R. (2001). fMR-adaptation: A tool for studying the functional properties of human cortical neurons. Acta Psychologica, 107(1-3), 293-321.

Gruber, T., \& Muller, M. M. (2005). Oscillatory brain activity dissociates between associative stimulus content in a repetition priming task in the human EEG. Cerebral Cortex, 15, 109-116.

Henson, R. (2001). Repetition effects for words and nonwords as indexed by eventrelated fMRI: A preliminary study. Scandinavian Journal of Psychology, 42 179-186.

Henson, R. (2003). Neuroimaging studies of priming. Progress in Neurobiology, $70(1), 53-81$

Henson, R., Mouchlianitis, E., Matthews, W., \& Kouider, S. (2008). Electrophysiological correlates of masked face priming. Neuroimage, 40(2), 884-895.

Henson, R., Shallice, T., \& Dolan, R. (2000). Neuroimaging evidence for dissociable forms of repetition priming. Science, 287(5456), 1269-1272.

Henson, R., Shallice, T., Gorno-Tempini, M. L., \& Dolan, R. J. (2002). Face repetition effects in implicit and explicit memory tests as measured by fMRI. Cerebra Cortex, 12(2), 178-186.

Horner, A. J., \& Henson, R. N. (2008). Priming, response learning and repetition suppression. Neuropsychologia, 46(7), 1979-1991.

Ishai, A., Pessoa, L., Bikle, P. C., \& Ungerleider, L. G. (2004). Repetition suppression of faces is modulated by emotion. Proceedings of the National Academy of Sciences of the United States of America, 101(26), 9827-9832.

James, T. W., \& Gauthier, I. (2006). Repetition-induced changes in BOLD response reflect accumulation of neural activity. Human Brain Mapping, 27(1), 37-46.

James, T. W., Humphrey, G. K., Gati, J. S., Menon, R. S., \& Goodale, M. A. (2000). The effects of visual object priming on brain activation before and after recognition. Current Biology, 10(17), 1017-1024.

James, T. W., Humphrey, G. K., Gati, J. S., Servos, P., Menon, R. S., \& Goodale, M. A (2002). Haptic study of three-dimensional objects activates extrastriate visua areas. Neuropsychologia, 40(10), 1706-1714.

Kim, S. J., \& Linden, D. J. (2007). Ubiquitous plasticity and memory storage. Neuron, 56(4), 582-592.

Kotz, S. A., Cappa, S. F., von Cramon, D. Y. \& Friederici, A. D. (2002). Modulation of the lexical-semantic network by auditory semantic priming: An event-related functional MRI study. Neuroimage, 17(4), 1761-1772.

Kouider, S., de Gardelle, V., Dehaene, S., Dupoux, E., \& Pallier, C. (2010). Cerebra bases of subliminal speech priming. Neuroimage, 49(1), 922-929.
Kouider, S., Dehaene, S., Jobert, A., \& Le Bihan, D. (2007). Cerebral bases of subliminal and supraliminal priming during reading. Cerebral Cortex, 17(9), 2019-2029.

Koutstaal, W., Wagner, A. D., Rotte, M., Maril, A., Buckner, R. L., \& Schacter, D. L. (2001). Perceptual specificity in visual object priming: Functional magnetic resonance imaging evidence for a laterality difference in fusiform cortex. Neuropsychologia, 39(2), 184-199.

Krekelberg, B., Boynton, G. M., \& van Wezel, R. J. A. (2006). Adaptation: From single cells to BOLD signals. Trends in Neurosciences, 29(5), 250-256.

Kumaran, D., \& Maguire, E. A. (2006). An unexpected sequence of events: Mismatch detection in the human hippocampus. PLoS Biology, 4(12), 2372-2382.

Kumaran, D., \& Maguire, E. A. (2009). Novelty signals: A window into hippocampal information processing. Trends in Cognitive Sciences, 13(2), 47-54.

Kuperberg, G. R., Lakshmanan, B. M., Greve, D. N., \& West, W. C. (2008). Task and semantic relationship influence both the polarity and localization of hemodynamic modulation during lexico-semantic processing. Human Brain Mapping, 29(5), 544-561.

Larsson, J., \& Smith, A. T. (2011). fMRI repetition suppression: Neuronal adaptation or stimulus expectation? Cerebral Cortex. Advance online publication. http://dx.doi.org/10.1093/cercor/bhr119.

Lau, H. C., Rogers, R. D., Haggard, P., \& Passingham, R. E. (2004). Attention to intention. Science, 303(5661), 1208-1210.

Li, L., Miller, E. K., \& Desimone, R. (1993). The representation of stimulusfamiliarity in anterior inferior temporal cortex. Journal of Neurophysiology, 69(6), 1918-1929.

Li, N., \& DiCarlo, J. J. (2008). Unsupervised natural experience rapidly alters invariant object representation in visual cortex. Science, 321(5895).

Loffler, G., Yourganov, G., Wilkinson, F., \& Wilson, H. R. (2005). fMRI evidence for the neural representation of faces. Nature Neuroscience, 8(10), 1386-1390.

Malach, R., Reppas, J. B., Benson, R. R., Kwong, K. K., Jiang, H., Kennedy, W. A., et al. (1995). Object-related activity revealed by functional magnetic-resonanceimaging in human occipital cortex. Proceedings of the National Academy of Sciences of the United States of America, 92(18), 8135-8139.

Marinkovic, K., Dhond, R. P., Dale, A. M., Glessner, M., Carr, V., \& Halgren, E. (2003). Spatiotemporal dynamics of modality-specific and supramodal word processing. Neuron, 38(3), 487-497.

McDermott, K. B., Ojemann, J. G., Petersen, S. E., Ollinger, J. M., Snyder, A. Z., Akbudak, E., et al. (1999). Direct comparison of episodic encoding and retrieval of words: An event-related fMRI study. Memory, 7(5-6), 661-678.

Mesulam, M. (2008). Representation, inference, and transcendent encoding in neurocognitive networks of the human brain. Annals of Neurology, 64(4), 367-378.

Miller, E. K., Li, L., \& Desimone, R. (1991). A neural mechanism for working and recognition memory in inferior temporal cortex. Science, 254(5036), 1377-1379.

Murray, S. O., \& Wojciulik, E. (2004). Attention increases neural selectivity in the human lateral occipital complex. Nature Neuroscience, 7(1), 70-74.

Nakamura, K., Dehaene, S., Jobert, A., Le Bihan, D., \& Kouider, S. (2007). Taskspecific change of unconscious neural priming in the cerebral language network. Proceedings of the National Academy of Sciences of the United States of America, 104(49), 19643-19648.

Orfanidou, E., Marslen-Wilson, W. D., \& Davis, M. H. (2006). Neural response suppression predicts repetition priming of spoken words and pseudowords. Journal of Cognitive Neuroscience, 18(8), 1237-1252.

Petit, J.-P., Midgley, K., Holcomb, P., \& Grainger, J. (2006). On the time course of letter perception: A masked priming ERP investigation. Psychonomic Bulletin $\mathcal{E}$ Review, 13(4), 674-681.

Race, E. A., Shanker, S., \& Wagner, A. D. (2009). Neural priming in human frontal cortex: multiple forms of learning reduce demands on the prefrontal executive system. Journal of Cognitive Neuroscience, 21(9), 1766-1781.

Rainer, G., \& Miller, E. K. (2000). Effects of visual experience on the representation of objects in the prefrontal cortex. Neuron, 27(1), 179-189.

Ranganath, C., \& D’Esposito, M. (2001). Medial temporal lobe activity associated with active maintenance of novel information. Neuron, 31(5), 865-873.

Ranganath, C. \& Rainer, G. (2003). Neural mechanisms for detecting and remembering novel events. Nature Reviews Neuroscience, 4(3), 193-202.

Rao, R. P., \& Sejnowski, T. J. (2002). Predictive coding, cortical feedback, and spiketiming dependent plasticity. In: R. P. Rao, B. A. Olshausen, \& M. S. Lewicki (Eds.), Perception and neural function (pp. 297-315). Cambridge, MA: MIT Press.

Raposo, A., Moss, H. E., Stamatakis, E. A., \& Tyler, L. K. (2006). Repetition suppression and semantic enhancement: An investigation of the neural correlates of priming. Neuropsychologia, 44(12), 2284-2295.

Ress, D., \& Heeger, D. J. (2003). Neuronal correlates of perception in early visual cortex. Nature Neuroscience, 6(4), 414-420.

Roediger, H. L., \& McDermott, K. B. (1993). Implicit memory in normal human subjects. In: F. Boller, \& J. Grafman (Eds.), Handbook of Neuropsychology (Vol. 8, pp. 63-131). Amsterdam: Elsevier.

Rossell, S. L., Price, C. J., \& Nobre, A. C. (2003). The anatomy and time course of semantic priming investigated by fMRI and ERPs. Neuropsychologia, 41(5), 550-564.

Rowe, J., Friston, K., Frackowiak, R., \& Passingham, R. (2002). Attention to action: Specific modulation of corticocortical interactions in humans. Neuroimage, 17(2), 988-998

Rugg, M. D., Otten, L. J., \& Henson, R. N. A. (2002). The neural basis of episodic memory: Evidence from functional neuroimaging. Philosophical Transactions of the Royal Society of London B, 357, 1097-1110. 
Salasoo, A., Shiffrin, R. M., \& Feustel, T. C. (1985). Building permanent memory codes-Codification and repetition effects in word identification. Journal of Experimental Psychology_General, 114(1), 50-77.

Salimpoor, V. N., Chang, C., \& Menon, V. (2010). Neural basis of repetition priming during mathematical cognition: Repetition suppression or repetition enhancement? Journal of Cognitive Neuroscience, 22(4), 790-805.

Schacter, D. L., \& Buckner, R. L. (1998). Priming and the brain. Neuron, 20(2), 185-195.

Schacter, D. L., Reiman, E., Uecker, A., Polster, M. R., Yun, L. S., \& Cooper, L. A. (1995). Brain-regions associated with retrieval of structurally coherent visual information. Nature, 376(6541), 587-590.

Schnyer, D. M., Ryan, L., Trouard, T., \& Forster, K. (2002). Masked word repetition results in increased fMRl signal: A framework for understanding signal changes in priming. Neuroreport, 13(3), 281-284.

Schott, B. H., Henson, R., Richardson-Klavehn, A., Becker, C., Thoma, V., Heinze, H. J., et al. (2005). Redefining implicit and explicit memory: The functional neuroanatomy of priming, remembering, and control of retrieval. Proceedings of the National Academy of Sciences of the United States of America, 102(4), 1257-1262.

Schott, B. H., Richardson-Klavehn, A., Henson, R., Becker, C., Heinze, H. J., \& Duzel, E. (2006). Neuroanatomical dissociation of encoding processes related to priming and explicit memory. Journal of Neuroscience, 26(3), 792-800.

Silver, M. A., Ress, D., \& Heeger, D. J. (2007). Neural correlates of sustained spatial attention in human early visual cortex. Journal of Neurophysiology, 97(1), 229-237.

Squire, L. R., Ojemann, J. G., Miezin, F. M., Petersen, S. E., Videen, T. O., \& Raichle, M. E. (1992). Activation of the hippocampus in normal humans-A functional anatomical study of memory. Proceedings of the National Academy of Sciences of the United States of America, 89(5), 1837-1841.

Summerfield, C., Trittschuh, E. H., Monti, J. M., Mesulam, M. M., \& Egner, T. (2008). Neural repetition suppression reflects fulfilled perceptual expectations. [Article]. Nature Neuroscience, 11(9), 1004-1006.

Summerfield, C., Wyart, V., Johnen, V. M., \& de Gardelle, V. (2011). Human scalp electroencephalography reveals that repetition suppression varies with expectation. Frontiers in Human Neuroscience, 5, 67.
Thoma, V., \& Henson, R. N. (2011). Object representations in ventral and dorsal visual streams: fMRI repetition effects depend on attention and part-whole configuration. Neuroimage, 57(2), 513-525.

Todorovic, A., Van Ede, F., Maris, E., \& de Lange, F. P. (2011). Prior expectation mediates neural adaptation to repeated sounds in the auditory cortex: An MEG study. Journal of Neuroscience, 31(25), 9118-9123.

Turk-Browne, N. B., Yi, D. J., Leber, A. B., \& Chun, M. M. (2007). Visual quality determines the direction of neural repetition effects. Cerebral Cortex, 17(2) 425-433.

van Turennout, M., Ellmore, T., \& Martin, A. (2000). Long-lasting cortical plasticity in the object naming system. Nature Neuroscience, 3(12), 1329-1334.

Vuilleumier, P., Schwartz, S., Duhoux, S., Dolan, R. J., \& Driver, J. (2005). Selective attention modulates neural substrates of repetition priming and "implicit" visual memory: Suppressions and enhancements revealed by fMRI. Journal of Cognitive Neuroscience, 17(8), 1245-1260.

Wagner, A. D., Shannon, B. J., Kahn, I., \& Buckner, R. L. (2005). Parietal lobe contributions to episodic memory retrieval. Trends in Cognitive Sciences, 9(9), 445-453.

Walker, M. P., \& Stickgold, R. (2006). Sleep, memory, and plasticity. Annual Review of Psychology, 57, 139-166.

Weisberg, J., van Turennout, M., \& Martin, A. (2007). A neural system for learning about object function. Cerebral Cortex, 17(3), 513-521.

Wible, C. G., Han, S. D., Spencer, M. H., Kubicki, M., Mh, N., Jolesz, F. A., et al. (2006) Connectivity among semantic associates: An fMRI study of semantic priming. Brain and Language, 97(3), 294-305.

Wig, G. S., Buckner, R. L., \& Schacter, D. L. (2009). Repetition priming influences distinct brain systems: Evidence from task-evoked data and resting-state correlations. Journal of Neurophysiology, 101(5), 2632-2648.

Wig, G. S., Grafton, S. T., Demos, K. E., \& Kelley, W. M. (2005). Reductions in neural activity underlie behavioral components of repetition priming. Nature Neuroscience, $8(9), 1228-1233$.

Wiggs, C. L., \& Martin, A. (1998). Properties and mechanisms of perceptual priming. Current Opinion in Neurobiology, 8(2), 227-233.

Yi, D. J., \& Chun, M. M. (2005). Attentional modulation of learning-related repetition attenuation effects in human parahippocampal cortex. Journal of Neuroscience, 25(14), 3593-3600. 